\title{
Colil: a database and search service for citation contexts in the life sciences domain
}

Toyofumi Fujiwara ${ }^{1}$ and Yasunori Yamamoto ${ }^{2^{*}}$

\begin{abstract}
Background: To promote research activities in a particular research area, it is important to efficiently identify current research trends, advances, and issues in that area. Although review papers in the research area can suffice for this purpose in general, researchers are not necessarily able to obtain these papers from research aspects of their interests at the time they are required. Therefore, the utilization of the citation contexts of papers in a research area has been considered as another approach. However, there are few search services to retrieve citation contexts in the life sciences domain; furthermore, efficiently obtaining citation contexts is becoming difficult due to the large volume and rapid growth of life sciences papers.
\end{abstract}

Results: Here, we introduce the Colil (Comments on Literature in Literature) database to store citation contexts in the life sciences domain. By using the Resource Description Framework (RDF) and a newly compiled vocabulary, we built the Colil database and made it available through the SPARQL endpoint. In addition, we developed a webbased search service called Colil that searches for a cited paper in the Colil database and then returns a list of citation contexts for it along with papers relevant to it based on co-citations. The citation contexts in the Colil database were extracted from full-text papers of the PubMed Central Open Access Subset (PMC-OAS), which includes 545,147 papers indexed in PubMed. These papers are distributed across 3,171 journals and cite 5,136,741 unique papers that correspond to approximately $25 \%$ of total PubMed entries.

Conclusions: By utilizing Colil, researchers can easily refer to a set of citation contexts and relevant papers based on co-citations for a target paper. Colil helps researchers to comprehend life sciences papers in a research area more efficiently and makes their biological research more efficient.

Keywords: Life sciences paper, Citation, Citation context, Co-citation, PMC Open Access Subset, RDF, SPARQL

\section{Background}

The ability to efficiently identify current research trends, advances, and issues in a research area is highly important to researchers to promote their research activities. For example, in cases of international collaborative research, researchers often need to read relevant papers and summarize the current knowledge about the research area beyond their own research fields [1]. Although review papers in the research area can suffice for this purpose in general, researchers are not necessarily able to obtain these papers at the right time from viewpoints of their interests. In addition, these papers reflect only previously published papers at the time of writing a

\footnotetext{
*Correspondence: yy@dbcls.rois.ac.jp

${ }^{2}$ Database Center for Life Science, Research Organization of Information and Systems, 178-4-4 Wakashiba, Kashiwa-shi, Chiba 277-0871, Japan

Full list of author information is available at the end of the article
}

review paper and viewpoints of its authors. To complement this issue, the utilization of the citation contexts for papers in a research area has been considered as another approach [2]. However, there are few search services to retrieve citation contexts in the life sciences domain, and to efficiently obtain the citation contexts for a target paper is becoming difficult due to the large volume and rapid growth of life sciences papers. Here, we introduce the Colil (Comments on Literature in Literature) database and a web-based search service called Colil for citation contexts in the life sciences domain.

In life sciences papers, citations are widely used and typically consist of two parts: a) a list of references found at the end of the citing paper that provides full bibliographic information for each source; and b) reference markers located in the text that are linked to the references [3]. The text surrounding a reference marker is defined as the 
citation context [4]; it contains information about the cited paper such as the important contributions of it, criticism against it, comparison of the work in it to the author's work, or use of the method described in it [5]. In previous studies, Bradshaw [6] showed that citation contexts provide many perspectives on a paper. Qazvinian and Radev [2] and Mei and Zhai [7] argued that citation contexts are useful for creating a summary of the important aspects of a paper. Furthermore, Elkiss et al. [8] and Divoli et al. [9] examined the relationships between the abstract and the citation contexts of a given life sciences paper, and their experiments showed that citation contexts tend to have additional and focused information that is not present in the abstract. These results indicate that citation contexts play an important role in representing the semantic content of life sciences papers.

To make better use of citation data, we built the Colil database as Linked Open Data (LOD) by using the Resource Description Framework (RDF). RDF is a promising technology for describing, publishing, and linking life sciences data on the Web [10]. In addition, LOD are linked to other related resources that use RDF and is released under an open license [11]. These technologies have the potential to facilitate data integration and provide the semantics to perform rich queries using the SPARQL query language [10]. To build the Colil database as LOD, we use a newly compiled vocabulary called Comments on Literature in Literature Ontology (COLILO) in addition to standard existing vocabularies such as Bibliographic Ontology (BIBO) [12], Dublin Core Metadata Initiative Metadata Terms (DCTERMS) [13], and Document Components Ontology (DoCO) [14]. We linked resources in the Colil database to their corresponding external ones such as Biotea [15], PubMed Central (PMC) [16], Digital Object Identifier (DOI) system [17], and TogoWS [18]. Although the Nature Publishing Group Linked Data Platform [19], the OpenCitations Project [20], and the Biotea Project [15] have already provided LOD that include citations, they have not provided citation contexts.

Our contribution is to provide LOD that include citation contexts in the life sciences domain. We provide three types of services that relate to the Colil database: a legacy web search interface (Colil), a SPARQL endpoint, and an ftp site to download dump files in RDF. The data needed for the Colil database have been extracted from open access papers deposited in the PubMed Central Open Access Subset (PMC-OAS) [16], which is made available under the Creative Commons License or similar licenses that generally provide users the rights to reuse and redistribute content. By using PMC-OAS, we can release the Colil database for reuse and redistribution under a Creative Commons License.

\section{Utility}

\section{Search service description}

Colil searches for a cited paper in the Colil database and then returns a list of the citation contexts for it and its relevant papers based on co-citations. Users can choose a search method (i.e., by PubMed ID or keywords). If a user initiates a search by typing a PubMed ID into the upper text box (Fig. 1a), Colil searches for a cited paper in the Colil database by this ID and then returns the corresponding citation contexts on the main page (Fig. 1c). If a user initiates a search by typing keywords into the lower text box (Fig. 1a), Colil searches for papers by using the PubMed search API [21]. Then, the search results are displayed in a modal dialog, where there is bibliographic information, including the title, authors, journal, year, volume, and issue (Fig. 1b). Each title is anchor text that links to the corresponding PubMed page. The user can choose one of the hit papers in the modal dialog, and then Colil returns the corresponding citation contexts on the main page (Fig. 1c). Colil's main page (Fig. 1c) is divided into four areas, described below:

1. Search condition area (the upper left) In the search condition area, Colil accepts a user query with a PubMed ID or keywords. For keywords, users can use search options that are available in PubMed, such as publication year and journal name, as in 'apoptosis 1995:2000[dp] "J Biol Chem" [jour]'.

2. Cited paper area (the upper right) In the cited paper area, Colil displays bibliographic information about the hit cited paper. This includes the title, authors, journal, year, volume, issue, and PubMed ID, and the title is anchor text that links to the corresponding PubMed page.

3. Citation context area (the lower right) In the citation context area, there is a list of the citation contexts for the hit cited paper. Colil highlights the reference markers, which are delimited by $>>$ and $<<$ in the citation contexts. Each row also includes the paper's title and the section title of the citing paper. Furthermore, there are links to the corresponding pages at Colil, PubMed, and PMC. Rows in the list can be sorted in ascending or descending order for one of the following factors: PubMed ID, paper title, and section title. The list shows 20, 40,60, or 100 results per page depending on the search condition. If the user clicks the anchor text for "Show query" on the upper side of the list, Colil shows the corresponding SPARQL query to obtain the result from our SPARQL endpoint. 
Identify a target paper

You can input a PubMed ID clirectly or via a PubMed search.

Input a PubMed ID

\section{PublMed ID here$$
\text { Try : } 10592235
$$

OR

\section{Input keywards}

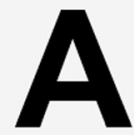

You can use search options such as a publication year $/ 2$ conditions like apoptosis 1995:2000[dp] "J Biolo of iPS 2006:2007[dp] "Cell"[jour]

\section{Try : iPS 2006:2008[dp] "Cell" [jour]}

Then, hit the enter key.

\begin{tabular}{l} 
Then, hit the enter key. \\
Colil by DBCLS \\
Identify a target paper \\
You can input a PubMod ID dirootly or via a \\
PubMed search. \\
Input a PubMed ID \\
\hline PubMod ID horo \\
\hline Try: 10592235 \\
OR \\
Input keywords \\
You can use search options such as a \\
publication year a journal conditions Ike \\
apoptosis 1995:2000[dp] "J Biol Chem" [jour] \\
\hline Keywords here \\
\hline Try : iPS $2006: 2008[d p]$ ' 'Cell "[jour] \\
Then, hit the enter key. \\
\hline
\end{tabular}

\section{Relevant Papers}

367 Induotion of pluripotent stem oells from mouse embryonic and adu fibroblast cultures by defined factors. Publfmod

365 Induced pluripotent stem cell lines derivod from human somatio oells. Publined

190 Embryonic stem coll lines derived from human blastocysts. Publiped

132 Goneration of gormline-eompetent induced pluripotent stem cells. Publined

127 Reprogramming of human somatic cells to pluripotency with defined factors. Publyidiod

92 In vitro reprogramming of fibroblasts into a pluripotont ES.
PubMed search result

Click the checkmark to choose a paper.

Query: iPS 2007:2008[dp] "Cell"[jour]

3 results found

\begin{tabular}{|l|l|}
$\leftarrow$ Previaus & Next $\rightarrow$ \\
\hline
\end{tabular}

$\checkmark$ Disease-specific induced plurip

Park IH, Arora N, Huo H, Maherali N, Ahfe T.S. amura A, Lensch MW, Cowan C

Hochedlinger K, Daley GQ

Cell 2008 Sep 5

Direct reprogramming of terminally differentiated mature B lymphocytes to

pluripotency.

Hanna J, Markoulaki S, Schorderet P, Carey BW, Beard C, Wernig M, Creyghton MP, Steine EJ, Cassady JP, Foreman R, Lengner CJ, Dausman JA, Jaenisch R

Cell 2008 Apr 18

$\checkmark$ Induction of pluripotent stem cells from adult human fibroblasts by defined factors. Takahashi K, Tanabe K, Ohnuki M, Narita M, Ichisaka T, Tomoda K, Yamanaka S 2007 Nov 30

\begin{tabular}{l|l|}
- Previaus & Next $\rightarrow$ \\
\hline
\end{tabular}

Fig. 1 Colil search at a glance. a Searching for papers with keywords by using the PubMed search API. By typing 'iPS 2007:2008[dp] "Cell" [jour]' into the lower text box and hitting the enter key, the user can search for papers by using the PubMed search API and keywords. b Choosing a hit paper. Here, the user is provided with three hits in the modal dialog. By clicking the paper titled, "Induction of pluripotent stem cells from mouse embryonic and adult fibroblast cultures by defined factors." Colil returns the corresponding results for the paper. c Displaying the search result. Here, the user is provided with the search result on the main page 
4. Relevant paper area (the lower left)

In the relevant paper area, there is a list of the relevant papers that are co-cited with the hit cited paper. Each row also includes a relevance score, the titles of relevant papers linking to the corresponding Colil page, and a link to the corresponding PubMed page. The relevance score is equal to the number of citing papers (Fig. 2). Rows in the list are sorted in descending order according to the relevance score, and only the top twenty relevant papers are displayed. If the user clicks the anchor text "Show query" on the upper side of the list, Colil shows the corresponding SPARQL query to obtain the result from our SPARQL endpoint.

\section{Example usage}

A researcher wants to identify target genes of a microRNA (miRNA) by using databases that collect manually curated miRNA-gene interactions with an experimental support. For this purpose, the researcher needs to identify current trends and issues concerning the databases. To obtain this information, by using Colil, the researcher can search for papers relevant to the databases and utilize the citation contexts for the papers. The researcher initiates a search for papers by typing keywords "database miRNA target interactions manually curated experimental support". As the result of the PubMed search (as of June 20th, 2015), Colil returns four papers, and three of them are original papers of widely-used databases such as TarBase, miRecords, and miRTarBase. Then, the researcher retrieves the corresponding citation contexts for the papers from Colil [see Additional file 1]; for example, the researcher can refer to the following citation contexts that help identify current trends and issues concerning the databases:

- Three databases are used to predict miRNA-target genes: TarBase (v6.0), miRecords (2013), and
miRTarBase (2013), which host the largest collection of manually curated experimental data;

- The experimentally validated miRNA-target interactions information have been documented in various databases, such as TarBase, MiRecords, miRWalk, miRTarBase, and miRNAMAP;

- TarBase and miRTarBase document the experimentally-verified information on miRNAtarget interactions along with their validation methods such as reporter genes, qPCR, western blotting, microarrays, proteomics, sequencing, and degradome sequencing data;

- For each miRNA, candidate targets have been inferred using data from six different databases (miRanda, PicTar, TargetScan, mirBase, miRTarget2, and TarBase) using the RmiR package from $\mathrm{R}$ Bioconductor (http://www.bioconductor.org/);

- Although miRTarBase has comprehensive information of miRNA targets from several organisms, it has scarce data on viral miRNA targets.

\section{Data retrieval}

In addition to Colil, we also provide a SPARQL endpoint that makes it possible for those users who develop their own applications to retrieve data in the Colil database. In practice, Colil retrieves and uses data from the Colil SPARQL endpoint. For instance, Table 1 shows a SPARQL query to retrieve a set of citation contexts for a target paper. In addition, Table 2 shows a SPARQL query to retrieve a title list of relevant papers for a target paper; the list is ordered by the relevance score.

\section{Construction and content Construction}

Figure 3 illustrates the process that we followed to build the Colil database. We obtained full-text papers from PMC-OAS. Then, we extracted citations (references and

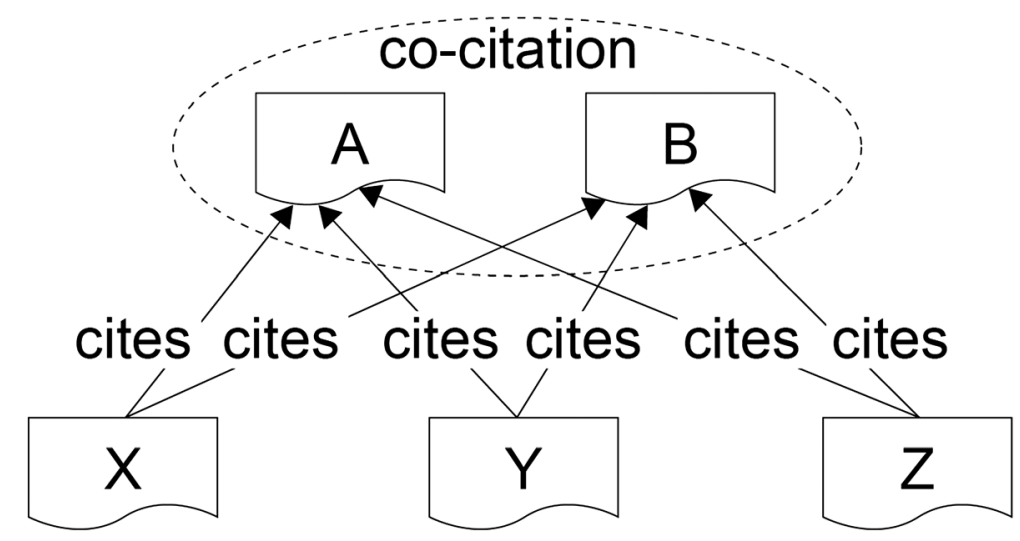

Fig. 2 Co-citation. In the case where the papers (A, B) are co-cited by three different papers $(X, Y, Z)$, the relevance score is 3 
Table 1 SPARQL query to retrieve a set of citation contexts for a target paper

\begin{tabular}{l}
\hline SPARQL query \\
\hline PREFIX colil: <http://purl.jp/bio/10/colil/ontology/201303\#> \\
PREFIX rdf: <http://www.w3.org/1999/02/22-rdf-syntax-ns\#> \\
PREFIX rdfs: <http://www.w3.org/2000/01/rdf-schema\#> \\
PREFIX togows: <http://togows.dbcls.jp/ontology/ncbi-pubmed\#> \\
select ?Context where \{ \\
[ rdf:value ?Context ; \\
colil:mentions [ \\
rdfs:seeAlso [ \\
rdf:type colil:PubMed; \\
togows:pmid "22135297" \\
] \\
].
\end{tabular}

reference markers) and citation contexts from these papers and counted co-citations from them. To build the Colil database, we used standard existing vocabularies, such as BIBO, DCTERMS, and DoCO, and compiled a new vocabulary. Below, we explain the construction method in more detail.

Table 2 SPARQL query to retrieve a title list of relevant papers for a target paper; the list is ordered by the relevance score

\begin{tabular}{l} 
SPARQL query \\
\hline PREFIX colil: <http://purl.jp/bio/10/colil/ontology/201303\#> \\
PREFIX rdf: <http://www.w3.org/1999/02/22-rdf-syntax-ns\#> \\
PREFIX rdfs: <http://www.w3.org/2000/01/rdf-schema\#> \\
PREFIX togows: <http://togows.dbcls.jp/ontology/ncbi-pubmed\#> \\
select ?RelevantPaperTitle ?score where \{ \\
[ rdfs:seeAlso [ \\
rdf:type colil:PubMed; \\
togows:pmid "22135297" \\
]; \\
colil:hasRelevantBibliographicResourceOf [ \\
colil:RelevantScore ?score; \\
colil:hasRelevantPaperld [ \\
rdfs:seeAlso [ \\
rdf:type colil:PubMed; \\
togows:ti ?RelevantPaperTitle \\
] \\
] \\
]. \\
\} order by desc(?score)
\end{tabular}

1. Obtaining full-text papers

We downloaded PMC-OAS papers, which are downloadable as XML files from the NCBI (National Center for Biotechnology Information) FTP site at ftp://ftp.ncbi.nlm.nih.gov/pub/pmc. In March 2014, there were 713,029 papers. From these PMC-OAS papers, we then selected 670,497 papers that are indexed in PubMed; this was so that we could provide users with the PubMed search function to look up papers. Finally, of these 670,497 papers, we selected 545,147 that cited at least one PubMed-indexed paper.

2. Identifying citations and citation contexts We parsed the XML files to extract references and reference markers. These components are easily identifiable because the files contain XML tags such as ref and xref. We then matched each bibliographic information component to its corresponding reference marker(s). Different journals utilize different formats for reference markers, and some reference markers are grouped together within a pair of parentheses; these multiple reference markers are concatenated by a connector (e.g., 1-6) or separated by a delimiter (e.g., 1,2,3,4,6). We therefore built a parser for the different formats and types of reference markers. We used PubMed-indexed citations only. Next, we identified citation contexts located around the reference markers. A citation context can be multiple sentences, a sentence, or a fragment of a sentence. Based on our preliminary survey of citation contexts, we first defined the citation context as being the sentence containing the reference marker. Then, if the reference marker was located at the end of the sentence, we added the next sentence to the citation context. Finally, if the number of characters from the start of the citation context to the reference marker was over 240 , only 240 characters before the reference marker and up to 240 characters after the reference marker were taken as the citation context.

3. Counting co-citations

If a pair of papers is cited by at least two papers, we counted it as a co-citation. For co-citations, the more the pair is cited by other papers, the more they are deemed to be semantically related [22].

4. Developing a newly compiled vocabulary We used BIBO, DoCO, and DCTERMS as standard vocabularies to facilitate inter-operability and cross-resource exploration. These three standard vocabularies can be used to describe citations and bibliographic information, the component parts of a 


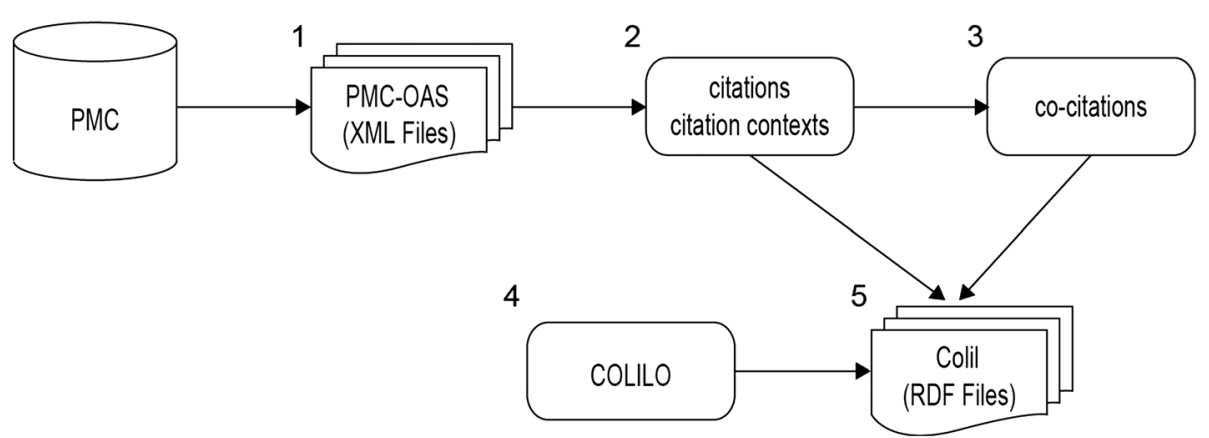

Fig. 3 Process of building the Colil database. The full-text papers of PMC-OAS were obtained from PMC (1). Citations and citation contexts were identified from the papers (2), and co-citations were counted from citations (3). A newly compiled vocabulary called COLILO was developed (4), and the Colil database was built by using COLILO, citations, citation contexts, and co-citations (5)

bibliographic document, and simple and generic resources, respectively. In addition, we compiled a new vocabulary called COLILO because the standard vocabularies were not comprehensive enough to express our data in RDF. Table 3 describes our new vocabulary consisting of six classes, four object properties, and three data type properties. To describe the vocabulary, we used the following font conventions: classes, object properties, and data properties. In addition, COLILO's Base URI is http://purl.jp/bio/10/colil/ontology/201303\#, and its prefix label is colil.

The ReferencePaper class and the CitationPaper class describe the cited paper and the citing paper, respectively. The citation context is described by the Context class, and the mention property links the citation context to the cited paper. The RelevantPaper class is used to describe the co-cited paper (i.e., relevant paper), and the cocitation With property, which has characteristics of symmetry, links the cited paper to the relevant paper. The RelevantBibliographicResource is used to describe the co-citation, which is between co-cited papers and has a relevance score using the RelevantScore property. The hasRelevantBibliographicResourceOf property links the cited paper to the co-citation, and the hasRelevantPaperId property links the co-citation to the relevant paper. The PubMed class and the Authors property are used to describe the bibliographic metadata and its authors, respectively.

Table 3 New vocabularies

\begin{tabular}{ll}
\hline Classes & $\begin{array}{l}\text { CitationPaper, Context, PubMed, ReferencePaper, } \\
\text { RelevantBibliographicResource, RelevantPaper }\end{array}$ \\
Object properties & $\begin{array}{l}\text { CocitationWith, hasRelevantBibliographicResourceOf, } \\
\text { hasRelevantPaperld, mentions }\end{array}$ \\
Data properties & Authors, pmcid, RelevantScore
\end{tabular}

The pmcid property is used to describe PMC IDs, which are identifiers of papers in PMC.

5. Building the Colil database

We built the Colil database by using COLILO.

Figure 4 shows an RDF graph that represents the relationship between the cited paper and the citing paper, which are represented as the colil:ReferencePaper and the colil:CitationPaper classes, respectively. We used the bibo:cites property to link the citing paper to the cited paper. We used the doco:contains property to represent a section in a document such as Introduction or Methods. Each section has a title and citation contexts, which are represented as the dcterms:title and the doco:contains properties, respectively. The citation context is represented as the colil:Context class, and the text is represented as the rdf:value property. We use the colil:mentions property to link the citation context to the cited paper.

Figure 5 shows an RDF graph that represents the relationship between the cited paper and a relevant paper. The colil:RelevantPaper class is used to represent the relevant paper, which is co-cited with another paper by other papers. We used the colil:cocitation With property to link the cited paper to the relevant paper. To represent a relevance score between the cited paper and relevant paper, we provided the co-citation, which is represented as the colil: RelevantBibliographicResource class. The co-citation includes the relevance score, which is represented as the colil:RelevantScore property. The colil:hasRelevantBibliographicResourceOf property links the cited paper to the co-citation, and the colil:hasRelevantPaperId property links the co-citation to the relevant paper.

Figure 6 shows an RDF graph that represents the bibliographic metadata, links to the 


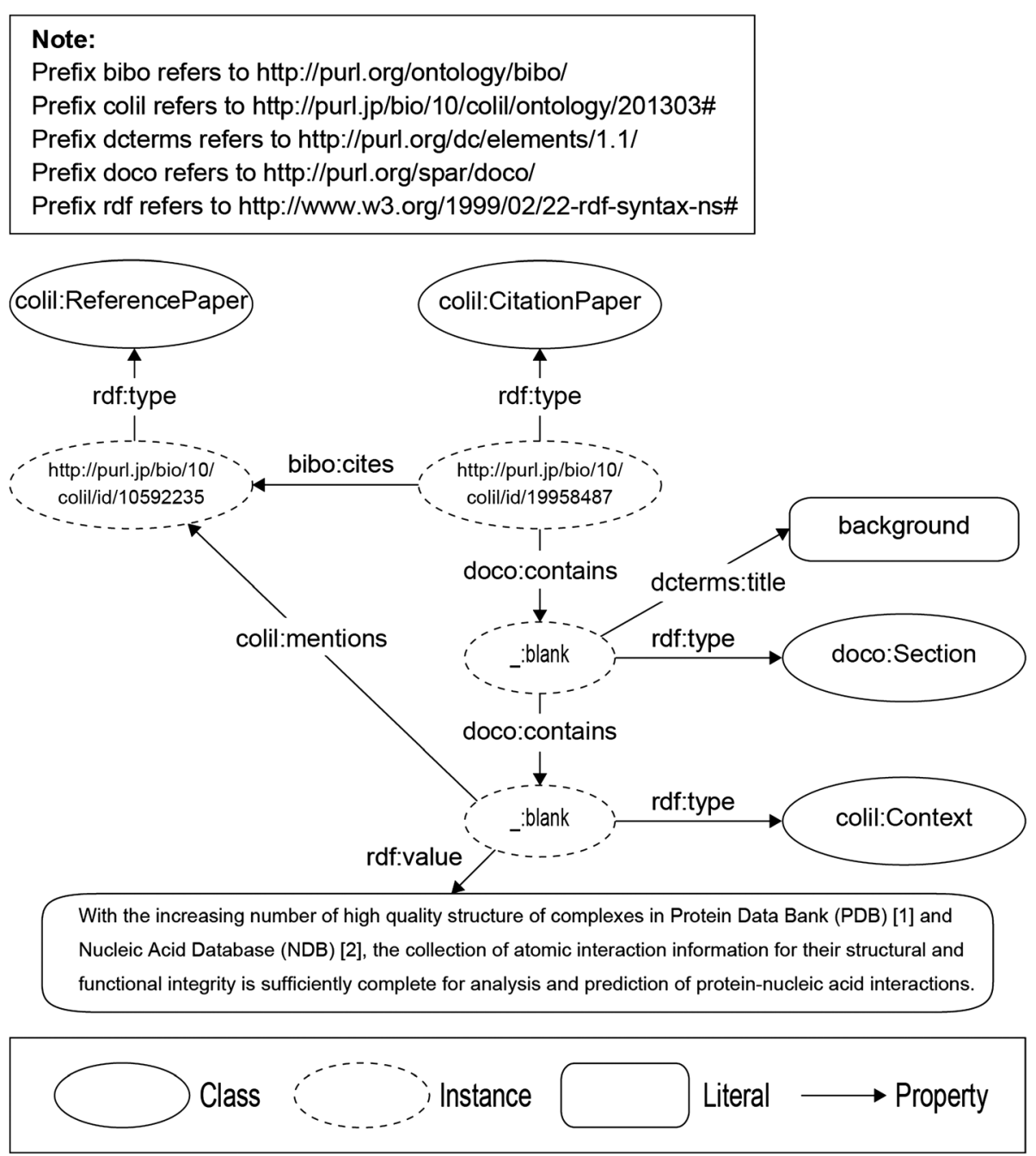

Fig. 4 RDF graph representing the relationship between the cited paper and the citing paper

external resources, and the paper identifiers. We used PMC IDs and DOIs so that users could easily find the original paper. They are represented as the colil:pmcid and bibo:doi properties, respectively. The rdfs:seeAlso property is used to link external resources such as PMC, Biotea, DOI system, and TogoWS. Bibliographic metadata are represented as the colil:PubMed class by using the rdfs:seeAlso property. We used the togows:pmid, colil:Authors, togows:ti, and togows:so properties to represent the bibliographic metadata of PubMed IDs, authors, title, and sources.

\section{Content}

Over the past 10 years, the number of PubMed-indexed papers published each year in PMC-OAS has grown, with the most recent years showing an exponential growth (Fig. 7). We obtained 545,147 PubMed-indexed PMC-OAS papers that cited at least one PubMed-indexed paper; the obtained papers were distributed across 3,171 journals. The papers contained 24,684,765 citation contexts, and each of them cited an average of 41.5 PubMed-indexed papers. Table 4 lists the top 20 journals ranked according to the number of the PMC-OAS papers contained in each journal.

Conversely, 5,136,741 PubMed-indexed papers have been cited by at least one PMC-OAS paper; the cited papers correspond to approximately one-quarter of the entire PubMed entries and are distributed across 11,588 journals. Table 5 lists the top 20 journals ranked according to the number of cited papers contained in each journal.

We found 27,832,062 co-citations in cited papers. To calculate the chances of a given paper having a co-cited paper in a different relevance score range, we classified 


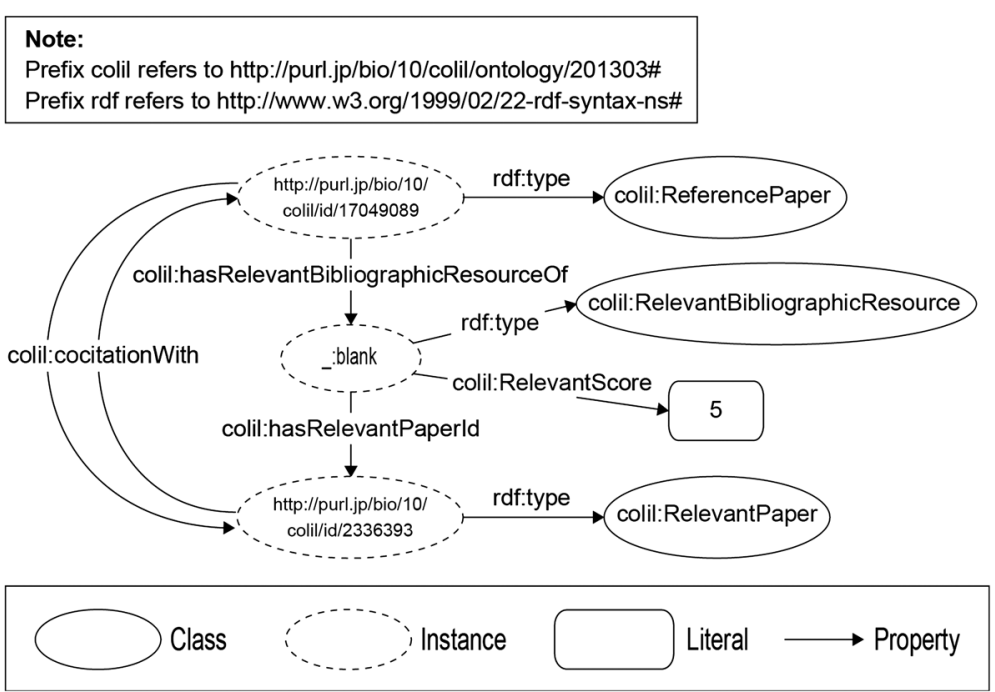

Fig. 5 RDF graph representing the relationship between the cited paper and the relevant paper

11 cases of co-citations according to their relevance scores (Table 6). Here, a chance is defined as the number of co-cited papers within the relevance score range divided by the total number of cited papers $(5,136,741)$. For example, there are $2,126,896$ co-cited papers with a relevance score of two and over, and the chance is approximately $41 \%$. Table 6 shows that this chance significantly decreases as the relevance score increases.

We released the Colil database, which consists of 445,671,312 triples, including approximately 6.8 million links to external resources. We use Virtuoso (VOS 7.1) [23] as the triple store; the Colil database is freely

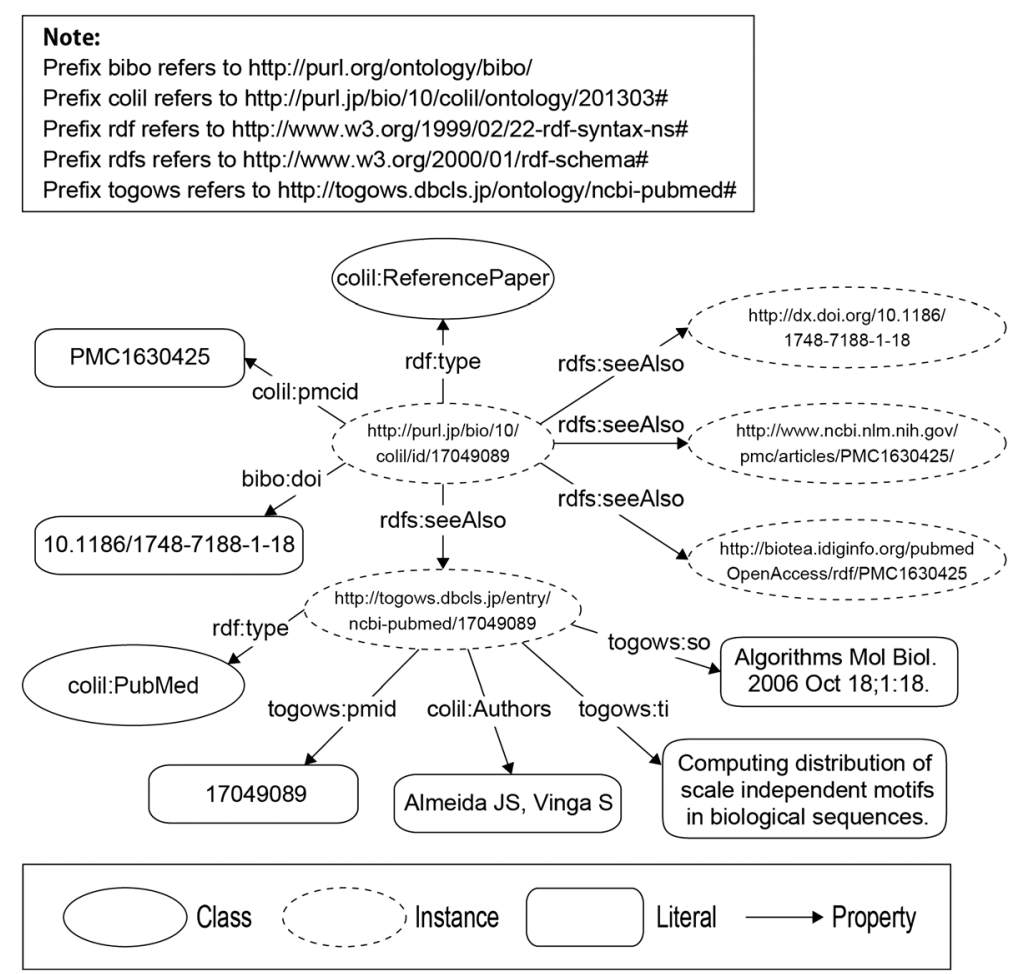

Fig. 6 RDF graph representing the bibliographic metadata, links to external resources, and paper identifications 


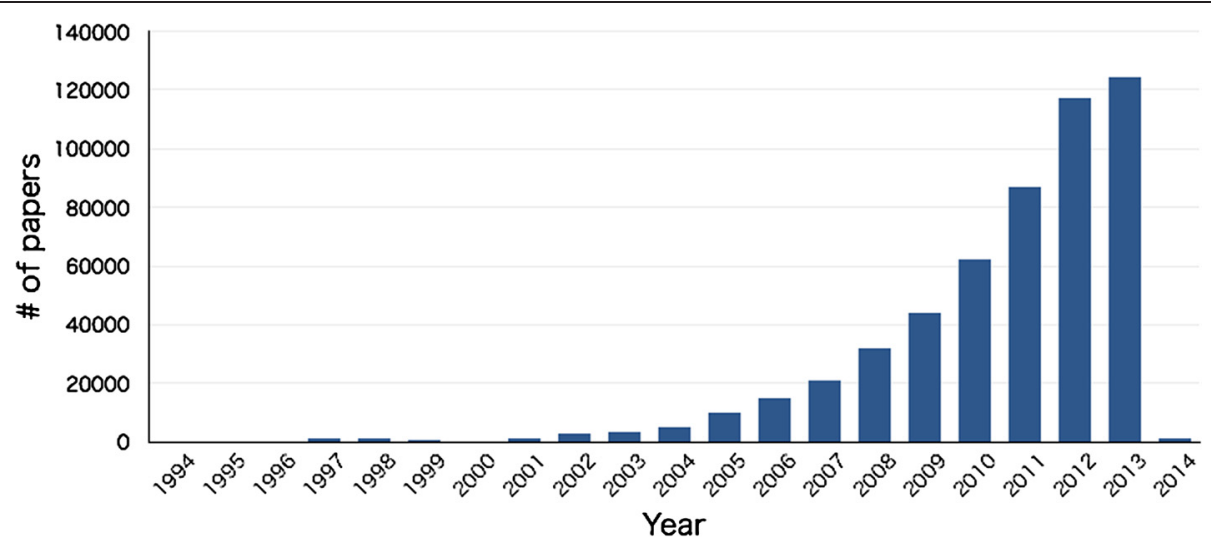

Fig. 7 Growth of the number of PubMed-indexed papers published per year in PMC-OAS from 1994 to 2014

available for querying and browsing through the SPARQL endpoint at http://colil.dbcls.jp/sparql. There is a faceted web service interface for the Colil database at http://colil.dbcls.jp/fct. You can also access and search the Colil database with a free text query without registration. Complete dumps of the RDF data are downloadable through the FTP site at $\mathrm{ftp}: / / \mathrm{ftp}$.dbcls.jp/colil; the data are available for reuse and redistribution under a Creative Commons Attribution 2.1 Japan License. We also provide a portal site at http://colil.dbcls.jp/portal

Table 4 Top 20 journals ranked according to the number of the PMC-OAS papers contained in each journal

\begin{tabular}{lr}
\hline Journal title & \# of papers \\
\hline PLOS ONE & 82735 \\
Nucleic Acids Research & 10660 \\
The Journal of Cell Biology & 6001 \\
BMC Public Health & 5791 \\
BMC Bioinformatics & 5771 \\
BMC Genomics & 5736 \\
Emerging Infectious Diseases & 5534 \\
The Journal of Experimental Medicine & 4536 \\
BMC Cancer & 4074 \\
PLOS Genetics & 3929 \\
International Journal of Molecular Sciences & 3809 \\
PLOS Pathogens & 3572 \\
Critical Care & 3359 \\
Scientific Reports & 3299 \\
Environmental Health Perspectives & 3210 \\
Evidence-based Complementary and & 2988 \\
Alternative Medicine: eCAM & 2965 \\
PLOS Computational Biology & 2731 \\
BMC Health Services Research & 2647 \\
Malaria Journal & 2618 \\
Journal of Medical Case Reports &
\end{tabular}

that includes an explanation of how to find the content and examples of querying the database by using the SPARQL endpoint.

\section{Discussion}

\section{Support for writing citation contexts}

As another use case, we envisage that Colil will help researchers to write citation contexts. In citation contexts, researchers must clarify the relationships between their paper and the papers cited within their paper [24].

Table 5 Top 20 journals ranked according to the number of cited papers contained in each journal

\begin{tabular}{lr}
\hline Journal title & \# of papers \\
\hline The Journal of Biological Chemistry & 95169 \\
Proceedings of the National Academy of & 69118 \\
Sciences of the United States of America & \\
PLOS ONE & 38722 \\
Journal of Immunology & 31637 \\
Nature & 27947 \\
Science (New York, N.Y.) & 27760 \\
Journal of Virology & 26123 \\
Biochemical and Biophysical Research Communications & 25379 \\
Cancer Research & 24396 \\
The Journal of Neuroscience & 23357 \\
Biochemistry & 23153 \\
Blood & 22681 \\
Journal of Bacteriology & 21325 \\
Nucleic Acids Research & 20585 \\
Biochimica et Biophysica Acta & 20309 \\
Lancet & 20246 \\
Brain Research & 18750 \\
Infection and Immunity & 16983 \\
Circulation & 16898 \\
The New England Journal of Medicine & 16467 \\
\hline
\end{tabular}


Table 6 The chances of a given paper having a co-cited paper in a different relevance score range

\begin{tabular}{cc}
\hline Relevance score range & Chance (\%) \\
\hline$\geq 2$ & 41.41 \\
$\geq 3$ & 19.68 \\
$\geq 4$ & 10.99 \\
$\geq 5$ & 6.88 \\
$\geq 6$ & 4.67 \\
$\geq 7$ & 3.35 \\
$\geq 8$ & 2.50 \\
$\geq 9$ & 1.93 \\
$\geq 10$ & 1.53 \\
$\geq 20$ & 0.32 \\
$\geq 50$ & 0.04 \\
\hline
\end{tabular}

However, this process may be difficult for some researchers, especially for non-English-speakers. In such cases, these researchers can learn how to write citation contexts more efficiently by observing the citation contexts that other researchers have made on a paper. We actually utilized Colil to write citation contexts in this paper and consider that Colil is suitable for this purpose. We continue to evaluate user experiences and will reflect the outcomes as Colil is developed further.

\section{Limitations of our approach}

Due to the limited number of citing papers in the Colil database, there are cases where Colil is not able to provide enough citation contexts for a target paper. For example, papers cited in five or fewer citation contexts account for approximately $76 \%$ of all the cited papers in the Colil database. One of the factors causing this issue is that the number of PubMed-indexed papers in PMC-OAS only corresponds to approximately $4 \%$ of total PubMed entries at present; however, we expect that this situation will gradually improve because open access publications are gaining popularity and becoming the norm [9]. Figure 7 shows the exponential growth of PMC-OAS papers over recent years, and the number of the PubMedindexed papers in PMC-OAS corresponds to approximately $14 \%$ of the entire PubMed entries published from 2010 to 2014.

In contrast, there were 11,086 papers that were cited in 100 or more citation contexts. Although Colil may provide users with enough information and perspectives for these papers, it can be time consuming to find appropriate citation contexts for references. To alleviate this issue, we are considering providing users with a function to narrow down citation contexts according to their purposes. In previous studies, Amjad et al. [5] and Teufel et al. [25] proposed 6 and 12 categories for citation purposes, respectively, and showed that they could classify citation contexts into categories with good accuracy by using their proposed approaches. For future work, we will consider annotating citation contexts based on their work.

\section{Comparison of Colil with Microsoft Academic Search}

As far as we know, Microsoft Academic Search (MAS) developed by Microsoft Research is the only search service to provide citation contexts in the life sciences domain except for Colil [26]. MAS is a freely accessible web-based search service and includes over 45 million records of academic publications. Although MAS has more indexes of the papers than does Colil, users cannot reuse and redistribute the data retrieved from it without previous permission. In addition, MAS has not been updated for about two years. On June 20th, 2015, we searched for the citation contexts at MAS and Colil for four papers retrieved with PubMed by using by the keywords "database miRNA target interactions manually curated experimental support" [Table 7]. MAS did not have the indexes of the papers that were published in or after 2013 since MAS was last updated in January 2013. The number of citation contexts for the papers is affected due to the lack of the citing papers that have been published in or after 2013. Colil was last updated on February 2nd, 2015 and has the indexes of four papers, and the number of citation contexts for each paper in Colil is higher than that in MAS. To keep up-to-date with the latest citing papers, we continue to update the Colil database twice a year.

\section{Problem of identifying citation contexts}

A citation context can be defined as sentences that comment on the work of a citing paper. Based on our preliminary survey of citation contexts, we defined a citation context as a sentence that contains a reference marker, except when the reference marker is located at the end of the sentence. We call a sentence that contains a reference marker an "explicit citing sentence" [5]. In this regard, some studies proposed methods to classify sentences as citation contexts from ones appearing around an explicit citing sentence [5]. Qazvinian and Radev [27] showed experimentally that they could retrieve important information to survey scientific papers from sentences

Table 7 Comparison of search results for cited paper

\begin{tabular}{|c|c|c|c|c|c|}
\hline \multirow[t]{2}{*}{$\begin{array}{l}\text { PubMed } \\
\text { ID }\end{array}$} & \multirow[t]{2}{*}{$\begin{array}{l}\text { Year of } \\
\text { publication }\end{array}$} & \multicolumn{2}{|c|}{$\begin{array}{l}\text { Cited paper } \\
\text { (Hit or miss) }\end{array}$} & \multicolumn{2}{|c|}{ \# of citation contexts } \\
\hline & & Colil & $\begin{array}{l}\text { Microsoft } \\
\text { academic search }\end{array}$ & Colil & $\begin{array}{l}\text { Microsoft } \\
\text { academic search }\end{array}$ \\
\hline 18996891 & 2009 & Hit & Hit & 220 & 42 \\
\hline 21071411 & 2011 & Hit & Hit & 158 & 3 \\
\hline 22135297 & 2012 & Hit & Miss & 88 & $\mathrm{~N} / \mathrm{A}$ \\
\hline 23376192 & 2014 & Hit & Miss & 1 & N/A \\
\hline
\end{tabular}


appearing around explicit citing sentences. To provide more comprehensive information, we are planning to improve our method of identifying a citation context based on their studies.

\section{Conclusions}

The Colil database includes citations, citation contexts, and co-citations extracted from full-text papers of PMCOAS. To help users to utilize the database in their respective research environments, we provide three types of services: a Colil search service, a SPARQL endpoint, and an $\mathrm{ftp}$ site. Colil can facilitate finding a set of citation contexts and relevant papers based on co-citations for a target paper. This should help researchers to comprehend life sciences papers in a research area more efficiently and make their biological research more efficient..

\section{Availability and requirements}

The Colil database is available under a Creative Commons Attribution 2.1 Japan License and can be downloaded at $\mathrm{ftp}: / / \mathrm{ftp} . \mathrm{dbcls.jp} /$ colil. COLILO is freely available under a Creative Commons Zero License at http://purl.jp/bio/10/ colil/ontology/201303. Colil is available for public access at http://colil.dbcls.jp/browse/papers/.

\section{Additional file}

Additional file 1: Retrieving sets of citation contexts for papers with PubMed ID 18996891, 21071411, 22135297, and 23376192. (XLSX $66 \mathrm{~kb})$

\begin{abstract}
Abbreviations
BIBO: Bibliographic Ontology; Colil: Comments on Literature in Literature; COLILO: Comments on Literature in Literature Ontology; DCTERMS: Dublin Core Metadata Initiative Metadata Terms; DoCO: Document Components Ontology; DOI: Digital Object Identifier; LOD: Linked Open Data; MAS: Microsoft Academic Search; miRNA: microRNA; PMC: PubMed Central; PMC-OAS: PubMed Central Open Access Subset; RDF: Resource Description Framework.
\end{abstract}

\section{Competing interests}

The authors declare that they have no competing interests.

\section{Authors' contributions}

YY originally conceived and designed this work. TF prepared the data implemented the services, and drafted the paper. All authors read and approved the final manuscript.

\section{Acknowledgements}

This work has been supported by the National Bioscience Database Center (NBDC) of the Japan Science and Technology Agency (JST). We thank Mr. Ryo Matsumiya for helping with the implementation of the Colil search service.

\section{Author details}

${ }^{1}$ INTEC Inc, 1-3-3 Shinsuna, Koto-ku, Tokyo 136-8637, Japan. ${ }^{2}$ Database Center for Life Science, Research Organization of Information and Systems, 178-4-4 Wakashiba, Kashiwa-shi, Chiba 277-0871, Japan.

Received: 19 January 2015 Accepted: 23 September 2015

Published online: 19 October 2015

\section{References}

1. Adams J. Collaborations: The fourth age of research. Nature. 2013:497(7451):557-60

2. Qazvinian V, Radev DR. Scientific paper summarization using citation summary networks. In: Proceedings of the 22nd International Conference on Computational Linguistics-Volume 1. Baltimore, USA: Association for Computational Linguistics; 2008. p. 689-96.

3. Masic I. The importance of proper citation of references in biomedical articles. Acta Informatica Medica. 2013;21(3):148.

4. Abu-Jbara A, Radev D. Reference scope identification in citing sentences. In: Proceedings of the 2012 Conference of the North American Chapter of the Association for Computational Linguistics: Human Language Technologies. Stroudsburg: Association for Computational Linguistics; 2012. p. 80-90.

5. Abu-Jbara A, Ezra J, Radev DR. Purpose and Polarity of Citation: Towards NLP-based Bibliometrics. Ann Arbor: HLT-NAACL; 2013. p. 596-606.

6. Bradshaw S. Reference directed indexing: Redeeming relevance for subject search in citation indexes. In: Research and Advanced Technology for Digital Libraries. Berlin Heidelberg: Springer; 2003. p. 499-510.

7. Mei Q, Zhai C. Generating Impact-Based Summaries for Scientific Literature Columbus, Ohio: ACL; 2008. p. 816-24

8. Elkiss A, Shen S, Fader A, Erkan G, States D, Radev D. Blind men and elephants: What do citation summaries tell us about a research article? J Am Soc Inf Sci Technol. 2008;59(1):51-62.

9. Divoli A, Nakov P, Hearst MA. Do peers see more in a paper than its authors? Advances in bioinformatics. 2012;2012:750214.

10. Jupp S, Malone J, Bolleman J, Brandizi M, Davies M, Garcia L, et al. The EBI RDF platform: linked open data for the life sciences. Bioinformatics. 2014;30(9):1338-9.

11. LinkingOpenData [http://www.w3.org/wiki/SweolG/TaskForces/ CommunityProjects/LinkingOpenData]

12. Bibliographic Ontology [http://purl.org/ontology/bibo/]

13. Dublin Core Metadata Initiative Metadata Terms [http://purl.org/dc/elements/1.1/]

14. Document Components Ontology [http://purl.org/spar/doco]

15. Castro LG, McLaughlin C, Garcia A. Biotea: RDFizing PubMed Central in support for the paper as an interface to the Web of Data. Journal of biomedical semantics. 2013;4 Suppl 1:S5

16. PMC [http://www.ncbi.n/m.nih.gov/pmc/]

17. Digital Object Identifier System [http://www.doi.org]

18. Katayama T, Nakao M, Takagi T. TogoWS: integrated SOAP and REST APIs for interoperable bioinformatics Web services. Nucleic Acids Res. 2010;38 suppl 2:W706-11.

19. Nature Publishing Group Linked Data Platform [http://developers.nature.com/ docs/read/linked_data]

20. OpenCitations Project [https://opencitations.wordpress.com/]

21. PubMed [http://www.ncbi.nlm.nih.gov/pubmed]

22. Small H. Co-citation in the scientific literature: A new measure of the relationship between two documents. J Am Soc Inf Sci. 1973;24(4):265-9.

23. OpenLink Software [http://www.openlinksw.com]

24. Agarwal S, Choubey L, Yu H: Automatically classifying the role of citations in biomedical articles. In AMIA Annual Symposium Proceedings. American Medical Informatics Association; 2010:11.

25. Teufel S, Siddharthan A, Tidhar D: Automatic classification of citation function. In Proceedings of the 2006 Conference on Empirical Methods in Natural Language Processing. Association for Computational Linguistics; 2006:103-110.

26. Microsoft Academic Search [http://academic.research.microsoft.com]

27. Qazvinian V, Radev DR: Identifying non-explicit citing sentences for citationbased summarization. In Proceedings of the 48th annual meeting of the association for computational linguistics. Association for Computational Linguistics; 2010:555-564. 